\title{
Modelo de la gestión del talento humano como desarrollo competitivo y sostenible, sector de Turismo en Santander, Colombia
}

Human resource management model as competitive and sustainable development: The tourism sector in Santander, Colombia

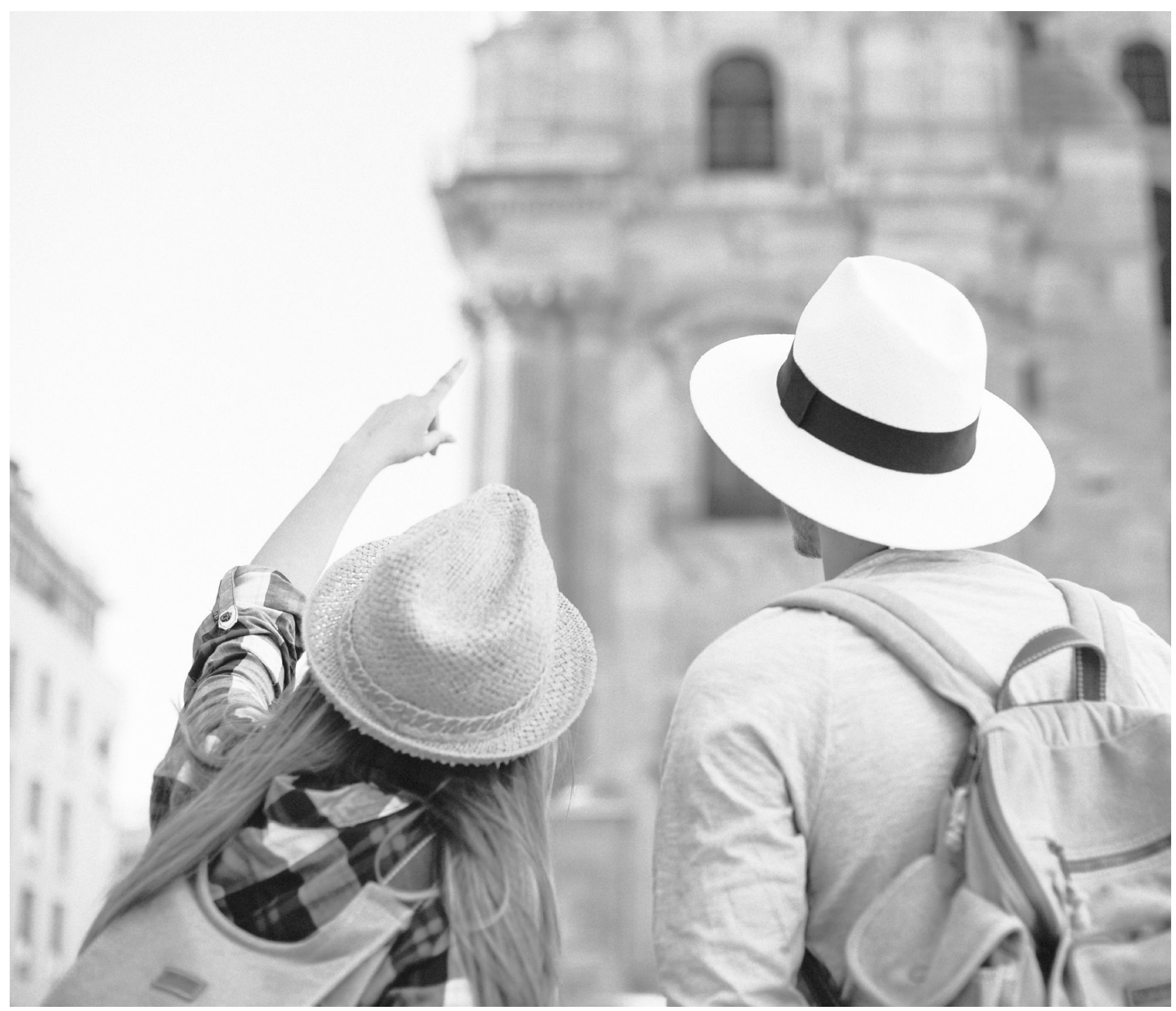




\title{
Modelo de la gestión del talento humano como desarrollo competitivo y sostenible, sector de Turismo en Santander, Colombia ${ }^{1}$ Human resource management model as competitive and sustainable development: The tourism sector in Santander, Colombia
}

\author{
Andrés Mauricio García Gómez², Claudya Mylleth Santana Franco ${ }^{3}$, Alba Patricia Guzmán Duque ${ }^{4}$
}

Artículo recibido en marzo 4 de 2020; artículo aceptado en mayo 10 de 2020

\begin{abstract}
Este artículo puede compartirse bajo la Licencia Creative Commons Atribución-NoComercial-Compartirlgual 4.0 Internacional y se referencia usando el siguiente formato: García, A., Santana, C. y Guzmán, A. (2020). Modelo de la Gestión del Talento Humano como desarrollo competitivo y sostenible, sector de Turismo en Santander, Colombia. I+D Revista de Investigaciones, 15 (2), 107-118. DOl: https://doi.org/10.33304/revinv.v15n2-2020010
\end{abstract}

\begin{abstract}
Resumen
La gestión del talento humano es una herramienta de dirección que se involucra en la estructura organizacional, con el fin de alcanzar los objetivos competitivos de la empresa. Es desde este horizonte de competitividad que surge la necesidad de utilizar un modelo de gestión del talento humano que marque una ruta estratégica y sostenible para la organización. En concreto, esta investigación busca determinar los factores que componen el modelo de gestión del talento humano para el sector turismo en el departamento de Santander, a partir de los modelos propuestos por Beer, Harper y Lynch, Chiavenato, Cuesta y Allen. Se utilizó una muestra de 59 mipymes que respondieron un instrumento en el nivel estratégico empresarial. Los resultados permitieron identificar el modelo de gestión del talento humano más apropiado para las mipymes del sector turismo. Se requiere mejorar el desarrollo de las competencias en el ámbito gerencial para buscar mayores niveles de competitividad y sostenibilidad para el desarrollo organizacional del sector.
\end{abstract}

Palabras clave: Competitividad, gestión del talento humano, sostenibilidad, turismo.

\footnotetext{
${ }^{1}$ Artículo de investigación, de tipo descriptivo y enfoque cualitativo, resultado de un proyecto de investigación terminado, perteneciente al área de ciencias sociales, sub área de competitividad y clima de negocios, desarrollado en el Grupo de Investigación en Ciencias Socioeconómicas y Empresariales (GICSE), financiado por las Unidades Tecnológicas de Santander (Bucaramanga, Colombia). Dirección: calle de los estudiantes n. ${ }^{\circ}$ 9-82, Ciudadela Real de Minas, PBX: (+57) 7 6917700. Línea gratuita: 018000940203 . Fecha de inicio: noviembre de 2016. Fecha de terminación: junio de 2018.

${ }^{2}$ Magíster en Dirección Estratégica de la Universidad Internacional Iberoamericana. Perteneciente al Grupo de Investigación en Ciencias Socioeconómicas y Empresariales, Unidades Tecnológicas de Santander, (Bucaramanga, Colombia). Dirección: calle de los estudiantes n. ${ }^{\circ}$ 9-82 Ciudadela Real de Minas, PBX: (+57) 7 6917700. Línea gratuita: 018000 940203. ORCID ID https://orcid.org/0000-0002-4988-2214. Correo electrónico institucional: agarcia@correo.uts.edu.co.

${ }^{3}$ Magíster en Dirección Estratégica de la Universidad Internacional Iberoamericana. Perteneciente al Grupo de Investigación en Ciencias Socioeconómicas y Empresariales, Unidades Tecnológicas de Santander, (Bucaramanga, Colombia). Dirección: calle de los estudiantes n. ${ }^{\circ} 9-82$ Ciudadela Real de Minas. PBX: (+57) 7 6917700. Línea gratuita: 018000 940203. ORCID ID: https://orcid.org/0000-0001-5435-6211. Correo electrónico institucional: claudiasantana@correo.uts.edu.co.

${ }^{4}$ Doctora en Gestión de las TIC, Universidad Politécnica de Valencia. Perteneciente al Grupo de Investigación en Ciencias Socioeconómicas y Empresariales, Unidades Tecnológicas de Santander (Bucaramanga, Colombia). Dirección: calle de los estudiantes n. ${ }^{\circ}$ 9-82, Ciudadela Real de Minas, PBX: (+57) 7 6917700. Línea gratuita: 018000 940203. ORCID ID https://orcid.org/0000-0003-1704-6884. Correo electrónico institucional: aguzman@correo.uts.edu.co.
} 


\begin{abstract}
Human talent management is a tool that involved in the organizational structure, used to achieve the competitive objectives of the company. It is from this horizon of competitiveness that the need to use a human talent management model arises, which marks a strategic and sustainable route for the organization. Specifically, this research seeks to determine the factors that make up the human talent management model for the tourism sector in the department of Santander, based on the models proposed by Beer Harper and Lynch, Chiavenato, Cuesta and Allen. Using a sample of 59 MSMEs, they answered an instrument at the strategic business level. The results allowed the identification of the most appropriate human talent management model for MSMEs in the tourism sector, where it is necessary to improve the development of competencies in the managerial field to seek higher levels of competitiveness and sustainability for the organizational development of the sector.
\end{abstract}

Keywords: Competitiveness, human talent management, sustainability, tourism.

\section{Introducción}

La importancia del turismo en la región santandereana es evidente en el crecimiento de unidades empresariales en diversos estilos de negocios, con un 5,8\% sobre el producto interno bruto (PIB) en el año 2017. Este crecimiento empresarial es ratificado por el Ministerio de Comercio, Industria y Turismo (MINCIT) (2018a), que afirma que el sector es favorable tanto en el ámbito local como en el nacional, en ventas de las agencias de viajes por un valor de 3600 millones USD para el año 2017. En Santander, la curva del sector es ascendente, pues en el año 2018 consolidó un crecimiento de ocupación, entre hoteles y restaurantes, del $31 \%$ (MINCIT, 2018b).

Precisamente, el turismo en Santander es pertinente para el crecimiento regional, con la integración de los municipios en una cadena de valor que ha logrado que el departamento sea identificado con un turismo de aventura, cultural y de negocios (Cámara de Comercio de Bucaramanga, 2019). Lo anterior ha permitido el crecimiento del $2,6 \%$ de la oferta laboral entre el mes de noviembre de 2017 y el mismo mes de 2018 (Compite 360, 2019). Desde otra perspectiva, el crecimiento del turismo ha sido jalonado por las inversiones en la infraestructura vial y en la construcción de carreteras de cuarta generación que han permitido el desarrollo entrelazado con los municipios del departamento, lo que ha facilitado la interacción entre las culturas regionales (MINCIT, 2018b).

Por otro lado, hoy en día las empresas obtienen una ventaja competitiva en el ciclo de aprendizaje de sus competencias y su gestión. Por tal motivo, hay que mantenerlas vitales y en continuo desarrollo, pues es un hecho que garantizan una constante renovación para una organización que aprende (Cuesta, 2010). De hecho, las organizaciones deben identificar cuáles son las competencias que requieren sus trabajadores, para fomentar espacios donde se desarrollen estrategias relacionadas con la gestión del conocimiento (Guzmán et al., 2017).
Esta investigación identificó el modelo de gestión del talento humano según la cultura regional organizacional, para adaptarse a los cambios económicos transversales que proporciona la globalización, y de esta manera buscar que las empresas sigan siendo competitivas y sostenibles en el mercado. Para cumplir lo anterior, se utilizó un instrumento de recolección de la información aplicado al nivel estratégico empresarial del sector de turismo en el departamento de Santander, a través del cual se identificó el modelo de gestión del talento humano para el sector turismo.

\section{Aproximación teórica}

El modelo de gestión del talento humano (MGTH) fundamenta su comprensión en el talento humano como fuente y denominador principal de los modelos de gestión. Desde este constructo del conocimiento, nace la impronta del desarrollo organizacional, en la que diversos autores presentan sus postulados sobre el talento humano. Chiavenato (2008), citado por Pico (2016), indica la diferenciación entre personas por medio de su capacidad competitiva, basándose en el conocimiento, habilidad, actitud y juicio ante diferentes procesos en la organización. Cadalzo (1996); Iris (1997); Menchetti y Flynn (1993); Mondy y Noe (2004), citados por Rodríguez-Durán y Murillo-Nevárez (2016), aseguran que el talento humano se enfoca en una gestión que se articula hacia un nivel estratégico-empresarial, en el que ellos pasan de ser agentes pasivos a agentes activos como administradores de todas las funciones organizacionales. Párraga (2016) afirma que la gestión del talento humano se debe enfocar hacia la estrategia, como una unidad dentro de la estructura que guía el proceso en la implementación de las estrategias y de los objetivos.

Por otra parte, el talento humano se va transformando en gestión del recurso humano (GTH), y está unido a los procesos productivos empresariales que proporcionan efectividad organizacional (Tejada, 2013). Macías y 
Aguilera (2012) afirman que se debe buscar otros matices diferenciadas al desarrollo tradicional, que solo se enfoca en el cargo dentro de la división del trabajo, lo que produce un retraso en la gestión del conocimiento. No obstante, Matošková (2012) lleva la gestión del talento humano a un proceso de actividades como herramientas para mejorar condiciones en lo cultural, reclutamiento adecuado, motivación más activa y desarrollo individual, a fin de mejorar las condiciones de trabajo.

Desde otra mirada, la gestión del talento humano se muestra a través de roles mediadores entre la capacidad de apropiarse del conocimiento y los resultados de los proyectos de la empresa, que asume sus actividades organizacionales en periodos medios y extensos (Popaitoon y Siengthai, 2014). Sánchez et al. (2015) asumen a la GTH como un ente mediador, pero en dirección a la gestión del conocimiento, para guiarlo hacia un comportamiento efectivo en todas sus funciones organizacionales, desde los niveles estratégicos, de modo que se permeabilice la estructura empresarial.

\section{Modelos de la gestión del talento humano}

\section{Modelo conceptual de Beer}

El modelo de Beer et al. (1984), citados por García, Úbeda y Lajarra (2015), se basa en cuatro políticas que son clave en la gestión del recurso humano. En estas la influencia de los empleados actúa como centro del modelo, lo que afecta las demás políticas (sistemas de trabajo, flujo de RH y sistemas de recompensa). De esta manera, el grupo social requiere observación permanente en función de sus motivaciones actitudes y necesidades (ver Figura 1).

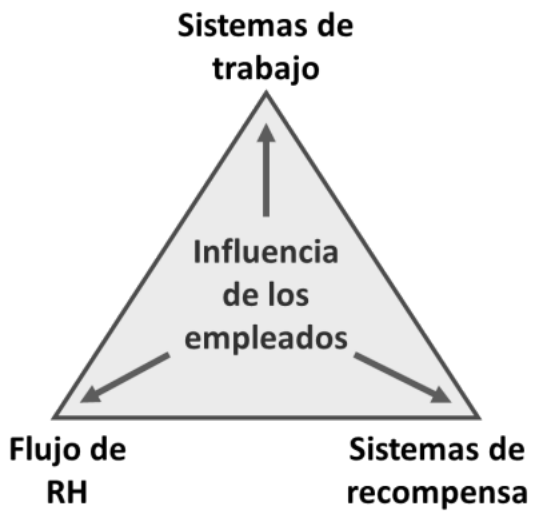

Figura 1. Modelo de gestión del recurso humano. Fuente: Beer (1989). Nota: El modelo inicial, donde el empleado es el centro. En él surgen las políticas de trabajo, las recompensas y el flujo de personal.

Lo anterior conduce a Beer (1989) a plantear una modificación de este modelo de manera conceptual, incluyendo en el proceso la auditoría de la GRH, centrada en la calidad como instrumento de retroalimentación al sistema; las técnicas e indicadores esenciales, lo que configura una tecnología para el diagnóstico; la proyección de la GRH como rectora del sistema en la dirección estratégica y la filosofía gerencial; y los factores de situación como base determinante al expresar que el hombre determina la conciencia y las políticas a definir en la GRH.

\section{Modelo de recurso humano de Harper y Lynch}

En 1992, Harper y Lynch, citados por Ramírez et al. (2018), presentan una serie de actividades fundamentales para la optimización de la gestión del recurso humano, en la que, a raíz de un plan estratégico, se realiza la previsión de las necesidades de la empresa. Este proceso se inicia con una relación del personal y la valoración del potencial humano, y finaliza con la auditoría, la optimización y el seguimiento. Todas estas actividades se interconectan y se orientan con la estrategia organizacional para conseguir los objetivos propuestos por la organización, como se observa en la Figura 2.

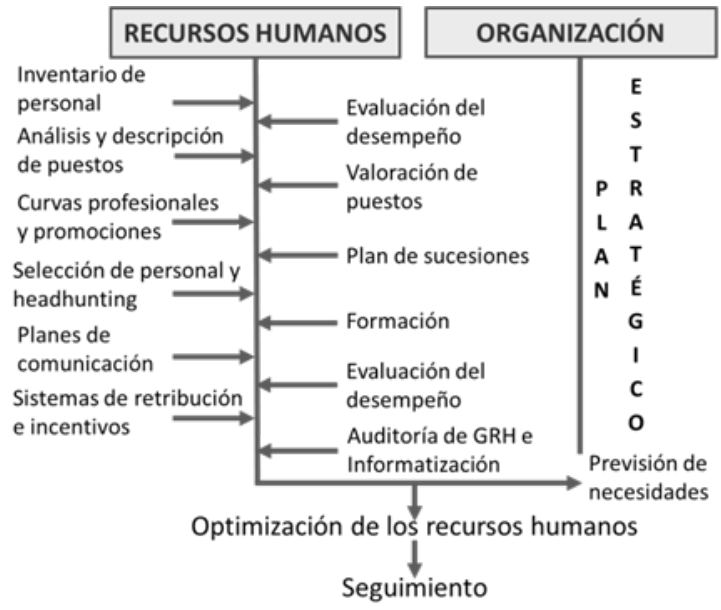

Figura 2. Modelo de recurso humano. Fuente: Harper y Lynch (1992). Nota: El modelo evidencia las actividades que se deben realizar en la gestión del recurso humano a partir de una planeación estratégica.

\section{Modelo de administración del recurso humano de Idalberto Chiavenato}

Chiavenato (2000) considera que la administración de recursos humanos permite la aplicación de todo el proceso administrativo, con el fin de conseguir un logro eficiente en su activo humano. Esto conduce hacia una exigencia para la búsqueda y permanencia de un grupo social comprometido en el logro de los objetivos organizacionales. Este modelo contempla cinco subsistemas que forman un proceso integral y eficiente, en el cual el personal es cautivado, y existen moderados y permanentes esquemas de control organizacional. Estos subsistemas se caracterizan por estar interrelacionados, pero a su vez son interdependientes, no se corresponden entre sí de una forma específica, son situacionales y 
dependen de factores de un entorno amable, estable y eficiente, entre otros, como se evidencia en la Figura 3.

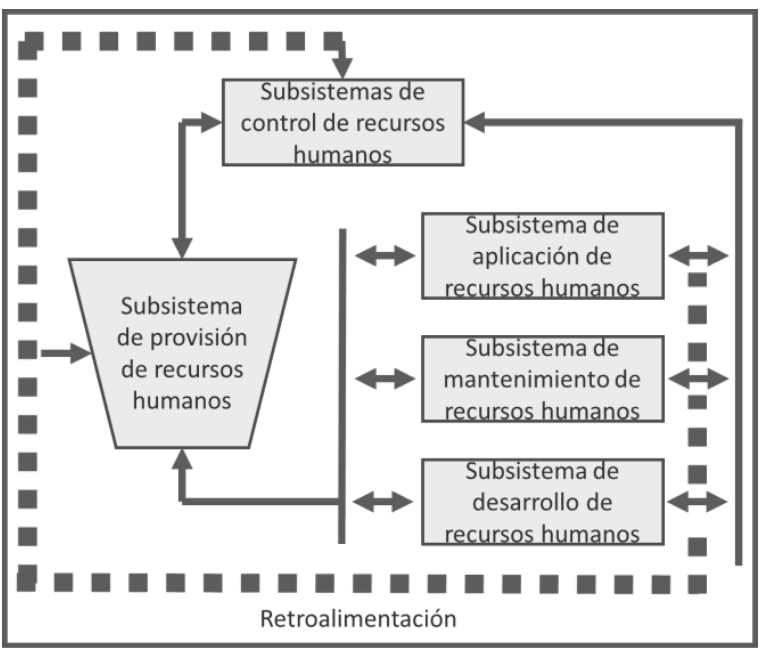

Figura 3. Modelo de administración de recursos humanos. Fuente: Chiavenato (2000). Nota: El modelo muestra la interrelación entre los cinco subsistemas que lo conforman.

\section{Modelo de recursos humanos de Cuesta}

Cuesta (2010) formula el modelo conceptual de recursos humanos, de análisis, influencia y control de gestión estratégica, con 6 enfoques (ver Figura 4):

1) Sistémico: La gestión del recurso humano es una cualidad que nace de la relación de sus procesos clave, de forma integral y fundamentada en el trabajo flexible, rechazando la teoría de Taylor y reconociendo la importancia del potencial humano en el desarrollo de sus multicompetencias.

2) Estratégico: Resalta la importancia de la proactividad y el accionar anticipado; se elimina la errónea costumbre de actuar después de que ocurre la dificultad, dado que se debe prever, para evitar que se presenten los problemas.

3) Participativo: Se acude a la ejecución de diferentes técnicas participativas con las que se brinde la posibilidad al personal de involucrarse en las actividades empresariales y toma de decisiones.

4) Interdisciplinario: El manejo del talento humano demanda incluir múltiples disciplinas científicas, e implica el accionar transfuncional respecto a todas las áreas.

5) De procesos: Considera la creación de valor inmerso entre el proveedor y el cliente con procesos enfocados en el cliente interno y externo de la organización.

6) De competencias laborales: Permite gestionar considerando las competencias laborales que poseen las personas que hacen posible el logro de los objetivos empresariales.

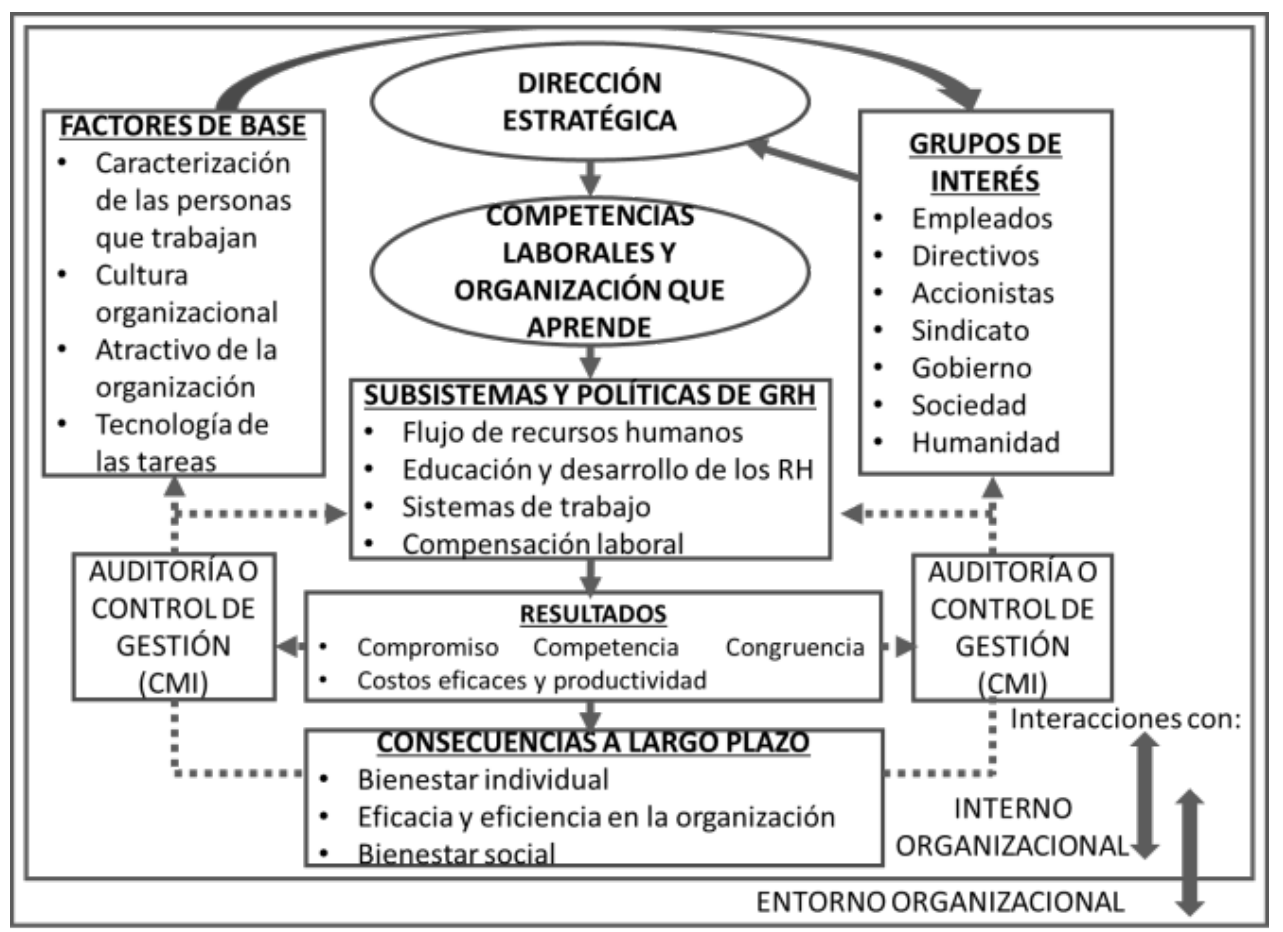

Figura 4. Modelo conceptual de recursos humanos de Cuesta. Fuente: Cuesta (2010). Nota: El modelo muestra la conexión entre el entorno externo con el entorno interno, mediante el desarrollo e interacción de los factores de base con los grupos de interés, los subsistemas y la auditoría de calidad. 
Este modelo se caracteriza porque tiene como fin y no como medio la educación y el crecimiento de las personas. Precisamente, ese es el centro del subsistema y de las políticas de recurso humano, flujo de personal, de educación y de desarrollo, de los sistemas de trabajo y de compensación laboral.

\section{Modelo de Gestión por Competencias de Alles}

Alles (2017), en su modelo (ver Figura 5), parte de la premisa "ser competitivos implica ser competentes", y trata de responder la pregunta " ${ }$ Es posible que un modelo de competencias alinee al grupo social empresarial con la estrategia para alcanzar la competitividad?". En este sentido, la autora afirma que la competitividad en una organización se alcanza al implementar la gestión por competencias.

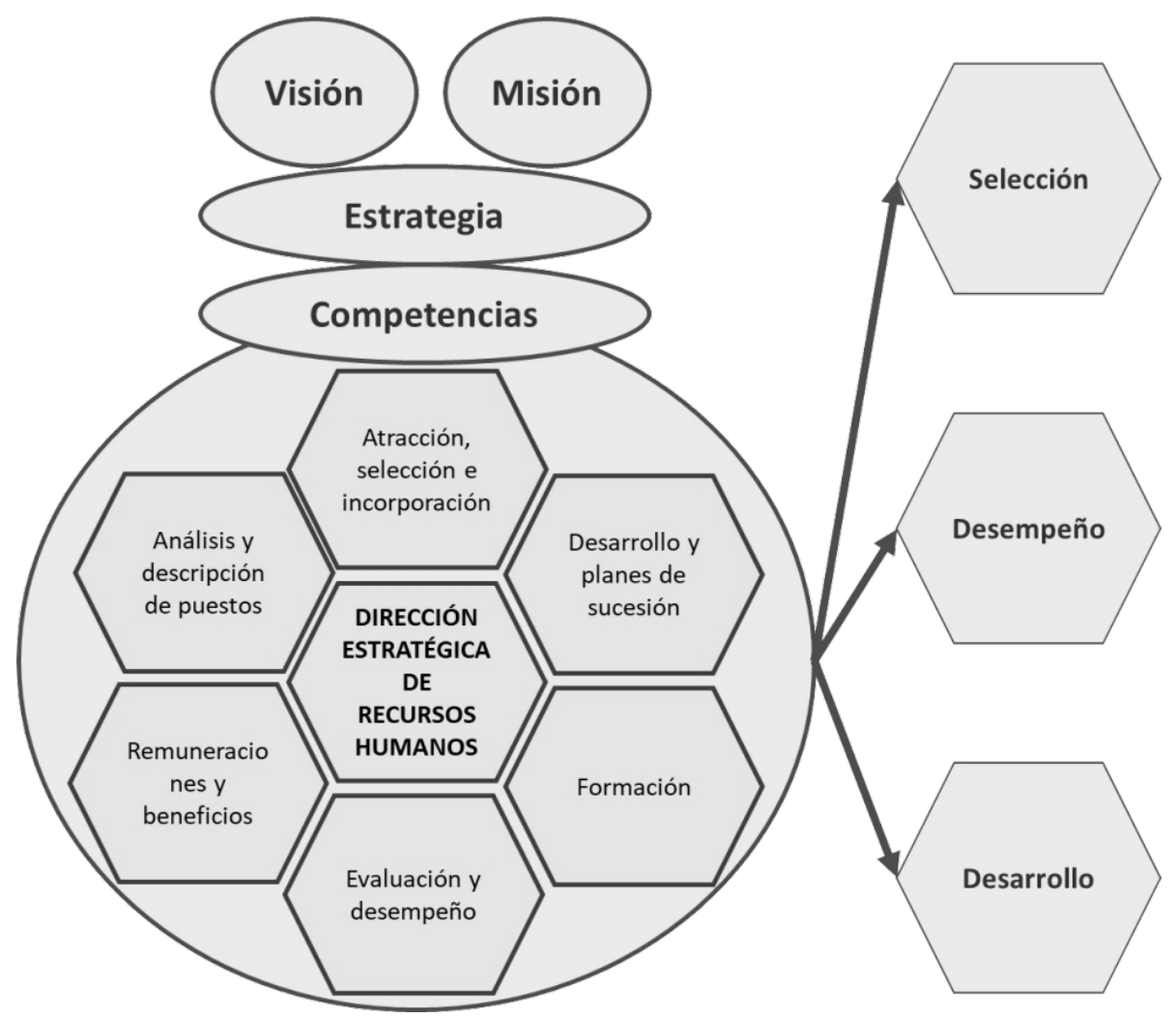

Figura 5. Modelo de gestión por competencias. Fuente: Alles (2017). Nota: El modelo evidencia que ser competitivos se logra al converger la misión y la visión de la empresa con los subsistemas de recursos humanos.

El modelo inicia desde la definición y actualización de la misión y la visión de la organización. Después se examinan y determinan los planes estratégicos y se precisan las competencias cardinales y específicas por cada área de la empresa. Posteriormente, se deben crear o modificar los subsistemas de recursos humanos: atracción, selección e incorporación, progreso y planes de formación, valoración de desempeño, salarios, beneficios, estudio y delineación de funciones.

\section{Modelo de administración de los recursos humanos de Werther y Davis}

Werther y Davis (2008) plantean el modelo de administración de recursos humanos como un sistema de muchas actividades interdependientes, en el que las actividades repercuten las unas en las otras, porque se encuentran estrechamente relacionadas entre sí (ver Figura 6). Por lo tanto, los autores especifican cinco subsistemas que están influenciados por los objetivos y las normas del área funcional de recurso humano y el factor externo de la organización:

1) Fundamentos y desafíos: El principal desafío es lograr el progreso de la eficiencia y la eficacia en la organización de una forma ética y responsable. Otros desafíos que se derivan son el crecimiento internacional de la actividad económica, el aumento de la competencia y las presiones del sector oficial. Para enfrentarlos, debe existir un engranaje en el departamento de recurso humano capaz de organizarse de manera que pueda alcanzar las metas y apoyar las labores de los gerentes operativos.

2) Preparación y selección: Implica tener un método de investigación sobre recursos humanos con datos actualizados de cada puesto y las necesidades futuras de personal. Esta información es indispensable para el diseño de los puestos de trabajo, con el fin de 
convertirlos en productivos y satisfactorios, y es útil para el requerimiento de personal a mediano y largo plazo.

3) Desarrollo y evaluación: Cada vez que ingresa personal nuevo a la empresa se realizan procesos de inducción en los que se dan a conocer las políticas e instrucciones de la empresa y del cargo que reciben, seguido de las capacitaciones para ser más productivos. Además, los empleados son calificados en su desempeño mediante evaluaciones para hacer retroalimentación y realizar acciones correctivas en los aspectos por mejorar.

4) Compensación y protección: La motivación para el recurso humano, en cierta parte, depende de una compensación equilibrada y justa en retribución a su labor ejecutada; pero aparecen dos escenarios adicionales: cuando los incentivos son insuficientes, se genera una alta en el movimiento de personal, y si el pago es muy alto, la empresa consigue desperdiciar su capacidad para competir.

5) Relación con el personal y la evaluación: El departamento de capital humano debe garantizar el bienestar del grupo laboral, crear y proteger sistemas de asesoría, mecanismos disciplinarios y de comunicación asertiva. Además, debe someterse a auditorías periódicas, para determinar su desempeño y generar nuevas y mejores formas de servir a la organización.

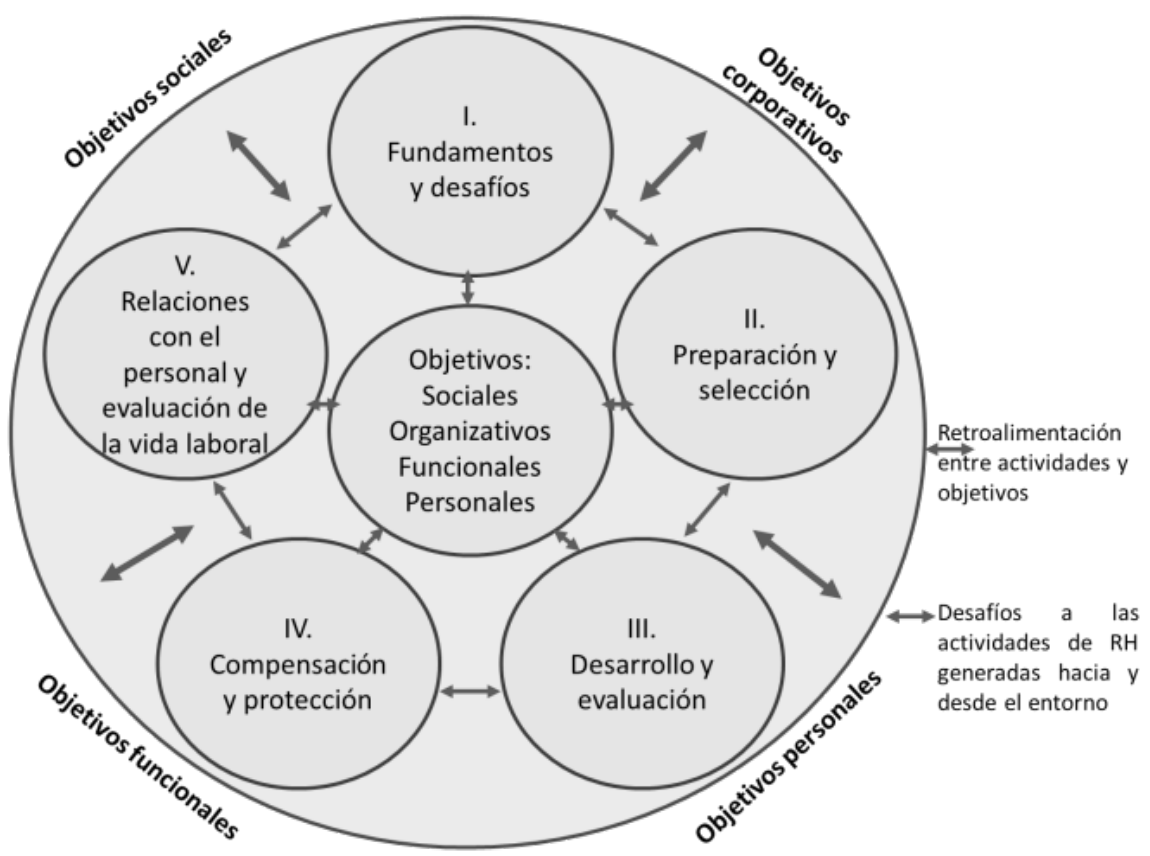

Figura 6. Modelo de administración de recursos humanos y subsistemas. Fuente: Werther y Davis (2008). Nota: En el modelo, las actividades de recursos humanos deben ser coherentes con los objetivos funcionales, personales, sociales y corporativos.

\section{Las competencias}

Las competencias se relacionan con el conjunto de las habilidades que requiere una persona o un trabajador para desempeñarse en su puesto de trabajo (Martin y Omrani, 2015). En este sentido, se requiere una serie de factores que se relacionen al tiempo para evidenciar que una persona es competente: debe poseer un conocimiento pertinente según su puesto de trabajo; tener la actitud para enfrentar diversos problemas; poseer los valores que le permitan ser ético en su quehacer y las habilidades para poner en práctica sus conocimientos en la resolución de un problema específico (Guzmán y Gutiérrez, 2018).

Existen diversas clasificaciones de las competencias. Para Muñoz-Cantero et al. (2014), una de ellas son las competencias genéricas, relacionadas con las habilidades que se necesitan para el desarrollo de las actividades de su puesto de trabajo, como aprender de su cargo, la solución de problemas, el trabajo con sus compañeros y la comunicación. Otras son las específicas, en las que el trabajador muestra su capacidad para la aplicación de las competencias anteriores y, por ende, para la resolución de problemas en un entorno competitivo. Es necesario indicar que una empresa puede ser competitiva si es capaz de ubicar su talento humano según las competencias que poseen y las necesidades de los puestos de trabajo, con lo que fomenta el aprender-aprendiendo (Guzmán y Gutiérrez, 2018).

Por otro lado, la serie guía número 21 del documento Ministerio de Educación Nacional (2016) determina seis competencias macrolaborales: intelectuales, personales, interpersonales, organizacionales, tecnológicas y empresariales para el emprendimiento. 
De estas competencias laborales se determinan las competencias gerenciales, concebidas por Saracho (2005), citado por Pradhan y Jena (2017), como la agrupación de conocimientos, destrezas y comportamientos en combinación con la actitud personal para el desempeño del rol gerencial.

\section{Metodología}

\section{Tipo de estudio}

La investigación tiene un alcance exploratorio y un enfoque cualitativo, mediante un análisis de la estructura organizacional de 59 mypimes del sector de turismo, para evidenciar el modelo de gestión del talento humano, dado que es este el objeto de estudio, y formular conclusiones fehacientes con base en la información recolectada (Fernández, 2017).

\section{Participantes}

La muestra la constituyen 59 mypimes de las 437 que existen en Santander (Colombia), clasificadas en agencias mayoristas, agencias operadoras y agencias de viajes y turismo (Confecámaras, 2016). Las mypimes investigadas fueron seleccionadas según su ubicación geográfica, registro y actividad económica, para indagar su comportamiento organizacional de la gestión del talento humano. La investigación se desarrolló en dos fases: el análisis situacional del sector de turismo, para conocer sus características generales y su desarrollo y enfoque gerencial, y la indagación sobre el comportamiento del nivel estratégico sobre el manejo de la gestión del talento humano, que permitió evaluar el nivel de competitividad y sostenibilidad del sector turismo en Santander.

\section{Materiales e instrumentos}

El instrumento utilizado para la recolección de la información fue un cuestionario con dos secciones: la primera parte consta de nueve (9) preguntas cerradas con opción de respuesta única, para caracterizar el talento humano; y la segunda, con ochenta y un (81) preguntas estructuradas en ocho (8) subcompetencias organizacionales, seleccionadas del documento del Ministerio de Educación Nacional de Colombia denominado "Articulación de la educación con el mundo productivo: capacidades laborales generales": liderazgo, comunicación, toma de decisiones, trabajo en equipo, resolución de conflictos, habilidades de negociación, actitud frente al cambio y motivación, encaminadas a alcanzar el cumplimiento de los objetivos. Las preguntas están diseñadas en la escala de Likert de 1 a 5 (donde 5 es igual a totalmente de acuerdo, y 1 es igual a totalmente en desacuerdo).

\section{Procedimiento}

El procedimiento fue abordado en 6 etapas:

Etapa 1. Diseño y elaboración del instrumento piloto: Para esta etapa se toma la investigación por medio de fuentes bibliográficas, para determinar las competencias gerenciales del nivel estratégico en las mipymes, así como los criterios y las instrucciones para su aplicación.

Etapa 2. Validación del instrumento: Se utilizó el instrumento preliminar, y el contenido fue validado por 5 pares investigadores expertos, utilizando para ello un formato que identificaba las características y competencias de la gestión del talento humano.

Etapa 3. Muestra piloto: Se tomó a un grupo de 15 funcionarios de mipymes del sector de turismo entre el nivel estratégico y táctico gerencial que cumplían con los criterios definidos.

Etapa 4. Creación del instrumento definitivo: Se analizaron las etapas anteriores, para unificar criterios de aplicación y determinar el instrumento final.

Etapa 5. Aplicación del instrumento: Se utilizó de acuerdo con los parámetros establecidos, y se aplicó a 59 mipymes del sector de turismo, ubicadas en la ciudad de Bucaramanga, direccionadas al nivel estratégico.

Etapa 6. Tabulación de la información: Para el análisis de los datos y la información obtenida, se utilizó la herramienta Statgrapchis.

\section{Resultados y discusión}

Los resultados iniciales se enfocan hacia un análisis situacional del sector de turismo en Santander, en el que se reconoce una básica gestión del talento humano, considerando sus características generales, su desarrollo y enfoque gerencial, y la tendencia del sector, puesto que solo sobrepasa la media porcentual en un $9 \%$, de las 437 empresas que poseen en Santander (Confecámaras, 2016). Lo anterior implica que tan solo un $59 \%$ de la muestra poblacional se interesa en preservar la dirección del GTH como un elemento principal para el desarrollo competitivo (ver Figura 7). En las demás mipymes del sector, se detectó que no se tiene especificada esta área funcional, aludiendo que la empresa es pequeña y que las actividades a desarrollar no ven pertinente la creación en estos momentos de esta área.

Para el sector de turismo, la premisa de inducción como proceso que permite un crecimiento integral en el grupo social empresarial y logra atraer y mantener la competitividad en la empresa (Álvarez, 2016) no funciona directamente. Primero se encuentran 
los procesos de inducción para nuevos y antiguos funcionarios que integran el sector de turismo, donde la creación del departamento de gestión de talento humano es deficiente; por ende, el proceso de inducción es incompleto: $\tan$ solo un $36 \%$ de las pymes lo hacen, lo que limita la eficiencia y eficacia en los procesos de la organización.

A estos resultados se unen otras áreas de la caracterización del sector turismo, como lo es el diseño de perfiles y el diagnóstico del requerimiento del personal para el cumplimiento de sus planes. Para Cuesta
(2019), el perfil empresarial es la base de la gestión del talento humano, junto con la conducta estratégica que permite un desempeño estable en todo el componente organizacional. Es desde esta perspectiva que el sector de turismo muestra deficiencia en el perfil y diagnóstico del personal, con valores porcentuales bajos, entre $37 \%$ y $17 \%$, respectivamente, y una estructura de la gestión de talento humano tan básica y casi inexistente que no permite reclutar un talento humano competitivo al nivel operativo organizacional. Lo anterior limita la toma de decisiones en el nivel estratégico de las pymes de turismo.

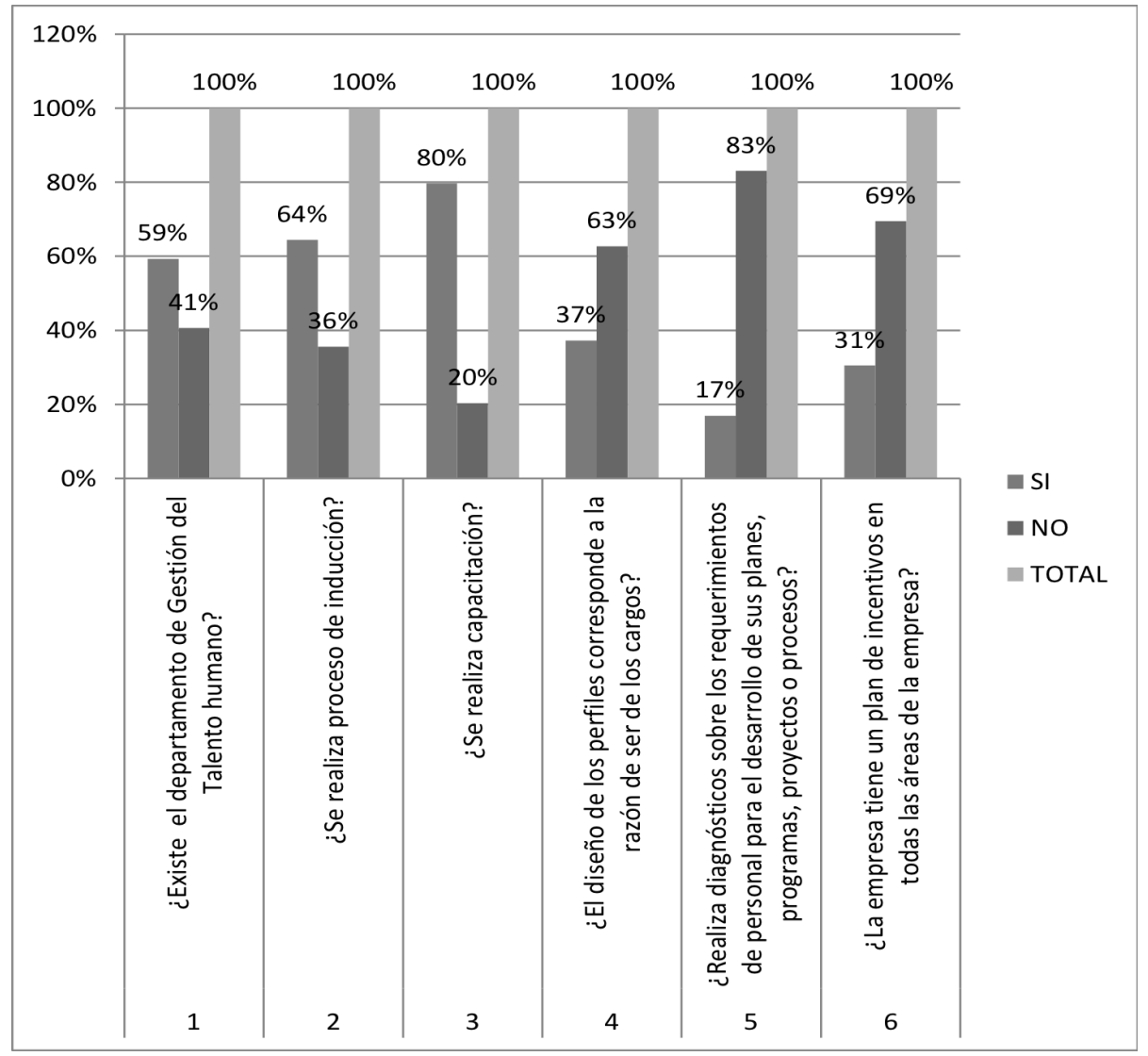

Figura 7. Se representan los resultados del análisis situacional que permite la caracterización de las pymes del sector de turismo. Fuente: Elaboración de los autores.

De otro lado, para determinar cuál es el modelo de gestión del talento humano más pertinente para el sector, se requiere conocer el grado de formación disciplinar del nivel estratégico. Para Cabrera y Arroyave (2019), el conocimiento y profesionalización de este nivel de la empresa es primordial para mantener el crecimiento y su sostenibilidad. En este aspecto, la investigación determinó resultados poco alentadores para este tipo de empresas de un sector que marca puntos de crecimiento en el ámbito nacional del $100 \%$. Se identificó una formación media profesional del $57 \%$ en niveles educativos entre profesionales y tecnólogos, de los cuales este último es el de menor porcentaje (42
\%). Estos valores son muy bajos para unificar criterios para un modelo de gestión del talento humano ideal para las empresas de turismo en Santander. Continuando con la escala de formación, se encuentran técnicos y bachilleres, con $43 \%$, donde el mayor valor porcentual es la formación media secundaria (24\%) (ver Figura 8). Esto demuestra la inestabilidad de las mipymes para alcanzar los niveles adecuados como empresas sólidas y sostenibles en el mercado. Para Cuesta (2010), el desarrollo de la gestión del talento humano se relaciona con las personas y su nivel de formación, que permite generar los subsistemas y políticas de un recurso humano, situación que no es identificada en las mipymes del sector de turismo. 


\section{El nivel de formación del gerente de la empresa es:}

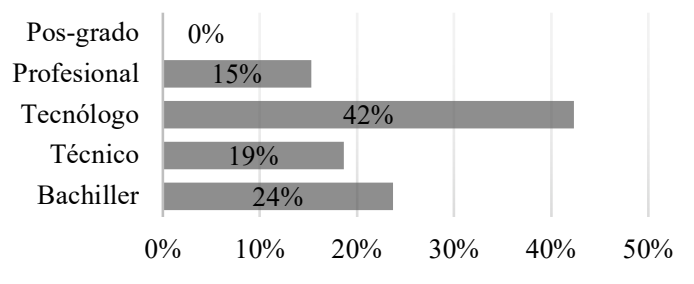

Figura 8. Formación escolar en el nivel estratégico de las empresas del sector de turismo en Santander. Fuente: Autores.

En la relación entre las competencias gerenciales con la experiencia, habilidad, conocimiento, actitud, motivación y valores, entre otros (Álvarez, 2017), se hace pertinente evidenciar que el sector de turismo requiere plantear un modelo de gestión del talento humano que identifique las competencias que interactúan como núcleo dinamizador que conduzca al talento humano a lograr los objetivos en todos los niveles organizacionales.

Desde esta perspectiva, se toman siete subcompetencias laborales gerenciales dentro del marco de las seis competencias labores del MEN, aplicadas en el nivel superior y nivel medio. Las competencias para evaluar son liderazgo, comunicación, toma de decisiones, resolución de conflictos, habilidades de negociación, actitud frente al cambio y motivación (ver Figura 9).

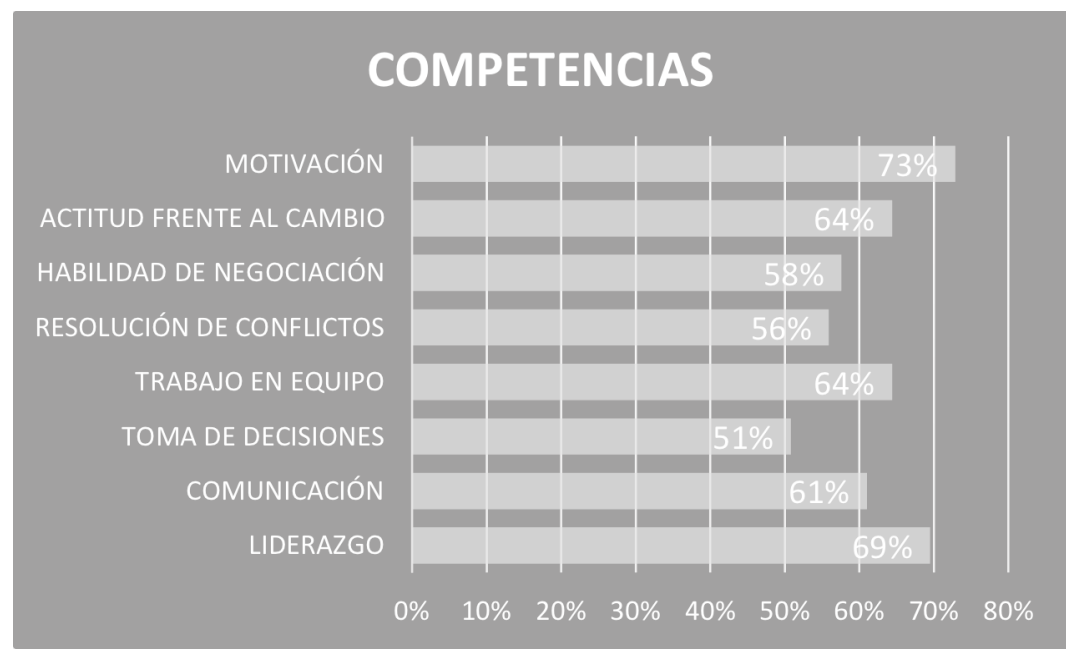

Figura 9. Competencias en escalas porcentuales de las competencias gerenciales ideales para un modelo de gestión del talento humano. Fuente: Autores.

Todas las competencias identifican un valor porcentual por encima de la media, desde $51 \%$ hasta un $73 \%$. Es preocupante la competencia de menor impacto porcentual, toma de decisiones, con el $51 \%$, dado que esta competencia está enmarcada en un nivel estratégico organizacional como fundamento de dirección que guiará la planeación de las mipymes del sector. Este aspecto se relaciona con el nivel de educación de los directivos del sector (ver Figura 8), donde se evidencia solo un $15 \%$ de formación profesional.

Le siguen en una escala inferior las competencias de habilidad de negociación y resolución de conflictos, con un $56 \%$ y $58 \%$, respectivamente. Estas competencias son fundamentales para el ejercicio empresarial. La primera hace parte esencial del sector, y su objeto social es la comercialización de servicios de turismo.

Las últimas competencias que muestran valores mayores, pero no muy alentadores son la actitud frente al cambio y el trabajo en equipo. Las dos comparten porcentajes mínimos por encima de la muestra porcentual (14\%), y son valores muy escasos que no permiten un desarrollo organizacional adecuado. Los porcentajes más altos se relacionan con las competencias de liderazgo (69 $\%)$ y motivación (73\%), lo que permite dar un parte de tranquilidad, porque ambas están unidas en el nivel estratégico.

Desde el análisis de los diferentes modelos de GTH, estas competencias se aproximan hacia el modelo de gestión por competencias de Alles (2017), en el que la GTH requiere mejor y mayor profundidad que logre unificar criterios según la cultura organizacional de este sector, y de esta manera mejorar la interacción entre las actividades elaboradas de las mipymes y quien las ejecuta, a fin de encaminarse hacia metas específicas organizacionales y mantener la sostenibilidad de la empresa.

\section{Conclusiones}

Las mipymes de turismo en Santander carecen de un área funcional de gestión del talento humano, situación que 
produce deficiencia en los procesos organizacionales y limita la sostenibilidad empresarial. Esto se debe al desconocimiento de la importancia de implementar un área funcional de gestión del talento humano, para desarrollar actividades básicas de la empresa, como el reclutamiento, la selección, la inducción y la capacitación. En este sentido, las empresas muestran inestabilidad en su desarrollo y descoordinación organizacional de su grupo social para la ejecución y desarrollo de actividades propias de las mipymes del sector de turismo.

La estructura predominante es la línea militar, porque se evidencian empresas que solo se esfuerzan en la consecución de objetivos comerciales, descuidando factores de mayor impacto para la empresa y que involucran a su grupo social. Esto limita la acción en actividades paulatinas y rutinarias propias de cargos netamente operativos, sin un esfuerzo para el cumplimiento de objetivos empresariales.

Por otra parte, los cargos no tienen manuales de funciones ni de procedimientos; una situación que conduce a realizar un proceso de contratación sin estimar perfiles del cargo, y contratando solo para el cumplimiento de actividades operativas con poca profundidad en los procesos. Lo anterior disminuye la eficiencia y la eficacia en la toma de decisiones, y centraliza toda la estructura organizacional en la unidad de mando única; esta es una característica propia de las empresas de línea militar (Münch, 2015).

Es importante indicar que las competencias gerenciales de mayor impacto son liderazgo y motivación. Este aspecto es muy positivo para las mipymes, considerando que en el nivel estratégico se requiere una guía especial que se base en estas dos competencias para el cumplimiento de las metas de la organización, y para mantener un ambiente laboral adecuado que se refleje en todos los lineamientos de autoridad y responsabilidad de las empresas de turismo. Ahora, la competencia gerencial de menor porcentaje es la toma de decisiones, y es algo negativo para el sector, puesto que la ausencia de esta competencia disminuye dos variables, acción y resultados, que se requieren para el desarrollo sostenible de las mipymes del sector de turismo, y que no se articulan en la velocidad de crecimiento que muestra el sector de turismo en Santander.

Finalmente, es necesario indicar que se ha identificado un modelo de gestión del talento humano que permite establecer unas competencias que se requieren para el nivel gerencial, y que debe adaptarse en las empresas de turismo para que se genere ventaja competitiva en el sector.

\section{Referencias}

Alles, M. A. (2017). Desempeño por competencias: evaluación de $360^{\circ}$. Buenos Aires: Ediciones Granica S.A.

Álvarez, H. (2016). Los procesos de inducción y capacitación en las IPS de Villavicencio [tesis de grado, Universidad de los Llanos]. Repositorio institucional, https://repositorio.unillanos.edu.co/ handle/001/888.

Álvarez, J. (2017). Competencias gerenciales y la gestión administrativa en instituciones educativas de la UGEL 04 [tesis de maestría, Universidad César Vallejo]. Repositorio Digital Institucional, http://repositorio. ucv.edu.pe/bitstream/handle/20.500.12692/8226/ Alvarez_PJA.pdf?sequence=1\&isAllowed=y.

Beer, M. (1989). Gestión de Recursos Humanos. Perspectiva de un director general. Texto y casos, Ed. Ministerio de Trabajo y Seguridad Social.

Beer, M., Spector, B. A., Lawrence, P. R., Mills, D. Q. y Walton, R. E. (1984). Managing Human Assets. Simon and Schuster.

Cabrera, S. L. y Arroyave, M. R. (2019). El nivel de profesionalización gerencial y su incidencia en la sostenibilidad en el tiempo de las empresas familiares. Revista Científica Ciencia y Tecnología, 19(23).

Cadalzo, D. (1996). Los perfiles de los puestos de trabajo: Base fundamental en la organización de la Gestión de los Recursos Humanos. Universidad Autónoma de México.

Cámara de Comercio de Bucaramanga. (2019). Clúster de Turismo. https://www.camaradirecta.com/fortalecermi-empresa/cluster/cluster-turismo/

Chiavenato, I. (2000). Gestión del Talento Humano. Mc Graw Hill.

Chiavenato, I. (2008). Gestión del Talento Humano. Mc Graw Hill.

Compite 360. (2019). Reporte sectorial. Actividad económica. http://www.compite360.com/sitio/adn/.

Confecámaras. (2016). Registro Nacional de Turismo, (RNT). http://rnt.confecamaras.co/

Cuesta, A. (2010). La gestión del talento humano y del conocimiento. Revista Latinoamericana de Psicología, 45(1), 157-160.

Cuesta, A. (2019). Tecnología de gestión de recursos humanos. Anales de la Academia de Ciencias de Cuba, 9(3).

Duque, A. P., \& Rojas, C. D. (2018). Las competencias digitales y el uso de las tecnologías sociales: el valor agregado en la comercialización en el sector calzado en Bucaramanga. I+D Revista de Investigaciones, 11(1), 17-26. https://doi.org/10.33304/revinv.v11n12018002

Rodríguez-Durán, M. y Murillo-Nevárez, S. (2016). Talento humano en la microempresa informal. Dominio de Las Ciencias, 2(2), 247-256. 
Fernández, R. (2017). Acerca de los enfoques cuantitativo y cualitativo en la investigación educativa cubana actual. Atenas, 2(34), 1-15.

García, F., Úbeda, M. y Lajarra, B. (2015). Estructura intelectual de la investigación sobre dirección de recursos humanos: un análisis bibliométrico aplicado a la revista The International Journal of Human Resource Management, 2000-2012. Revista Europea de Dirección y Economía de la Empresa, 24(3), 149-161.

Guzmán, A., Oliveros, D. y Mendoza, M. (2017). Scientific Competencies: A Mechanism to Favour the Inclusion of Working Market Professionals. Journal of Baltic Science Education, 16(2), 175-187.

Guzmán, A. P. y Gutiérrez, C. D. (2018). Las competencias digitales y el uso de las tecnologías sociales: El valor agregado en la comercialización en el sector calzado en Bucaramanga. I+D Revista de Investigaciones, 11(1), 17-26. https://doi.org/10.33304/revinv.v11n12018002

Harper, S. y Lynch, J. (1992). Manuales de recursos humanos. Ed. Gaceta de los Negocios.

Iris, P. (1997). Sistema de Gestión de Recursos Humanos del Centro Nacional de Sanidad Agropecuaria.

Macías, C. y Aguilera, A. (2012). Contribución de la gestión de recursos humanos a la gestión del conocimiento. Estudios Gerenciales, 28(123), 133-148. https://doi. org/10.1016/S0123-5923(12)70209-7

Martin, L. y Omrani, N. (2015). An Assessment of Trends in Technology Use, Innovative Work Practices and Employees' Attitudes in Europe. Applied Economics, 47(6), 623-638. https://doi.org/10.1080/00036846.2 014.978072

Matošková, J. (2012). How to Support Knowledge Management Through Human Resource Management Activities. International Journal of Knowledge, Culture and Change Management, 11(3), 207-222.

Menchetti, B. Y. y Flynn, C. C. (1993). Empleo con apoyo. Nuevas direcciones para la evaluación profesional. Siglo Cero, 25(148), 5-19.

Ministerio de Comercio, Industria y Turismo. (2018a). Resultados para el turismo para el año 2018. https://www.mincit.gov.co/getattachment/estudioseconomicos/estadisticas-e-informes/informes-deturismo/2018/oee-vt-turismo-2018-28-03-2019.pdf. aspx.

Ministerio de Comercio, Industria y Turismo. (2018b). Ocupación hotelera por departamento. https://www. mincit.gov.co/estudios-economicos/estadisticas-einformes/sistemas-de-estadisticas-de-consulta-rapida/ estadisticas-economicas-intercambiables-indice.

Ministerio de Educación Nacional (2016). Guía No. 21 Competencias laborales https://www.mineducacion. gov.co/1759/w3-printer-106706.html

Mondy, R. W. y Noe, R. M. (2004). Administración de recursos humanos. Pearson Educación.
Münch, L. (2015). Adminsitración. Proceso administrativo clave del éxito empresarial. Pearson.

Muñoz-Cantero, J., Rebollo, N. y Espiñeira, E. M. (2014). Percepción de competencias en el EEES: análisis en el Grado de Educación Primaria. Revista Electrónica Interuniversitaria de Formación Del Profesorado, 17(3), 123-139. https://doi.org/10.6018/reifop.17.3.204091

Párraga, C. (2016). Gestión del talento humano en la universidad ecuatoriana actual. Revista Didasc@lia: Didáctica y Educación, 7(6), 285-290.

Pico, L. (2016). La gestión del talento humano, recurso indispensable para la organización en el entorno competitivo actual. INNOVA Research Journal, 1(11), 97-104. https://doi.org/10.33890/innova.2016.122

Popaitoon, S. y Siengthai, S. (2014). The Moderating Effect of Human Resource Management Practices on the Relationship Between Knowledge Absorptive Capacity and Project Performance in ProjectOriented Companies. International Journal of Project Management, 32(6), 908-920.

Pradhan, R. K. y Jena, L. K. (2017). Employee Performance at Workplace: Conceptual Model and Empirical Validation. Business Perspectives and Research, 5(1), 69-85.

Ramirez, A., Pérez, A., Hernández, I. y Laguna, O. (2018). Enfoque estratégico de la planeación de los recursos humanos. Visión teórico práctico en el sector empresarial. Estudios del Desarrollo Social: Cuba y América Latina, 6(1), 133-144.

Sánchez, A. A., Marín, G. S. y Morales, A. M. (2015). The Mediating Effect of Strategic Human Resource Practices on Knowledge Management and Firm Performance. Revista Europea de Dirección y Economía de la Empresa, 24(3), 138-148. https://doi. org/10.1016/j.redee.2015.03.003

Saracho, J. (2005). Un modelo general de gestión por competencias. Modelos y metodologías para la identificación y construcción de competencias. http://libritosfavoritos.blogspot.pe/2012/10/un$\% 0$ Amodelo-general-degestion-por.html

Tejada, A. (2013). Los modelos actuales de gestión en las organizaciones. Gestión del talento, gestión del conocimiento y gestión por competencias. Piscología desde el Caribe, 115-133.

Werther, B. y Davis, K. (2008). Administración de recursos humanos. McGraw-Hill Interamericana. 